\title{
Delayed Septal Perforation as a Complication of COVID-19: A Case Report
}

\author{
Corinne Wee $^{1} \cdot$ Joseph Younis $^{1} \cdot$ Thomas Xia $^{1} \cdot$ Bahman Guyuron $^{2}$
}

Received: 25 August 2020/Accepted: 26 November 2020/Published online: 5 January 2021

(C) Springer Science+Business Media, LLC, part of Springer Nature and International Society of Aesthetic Plastic Surgery 2021

\begin{abstract}
Background There are increasing reports of the effects of SARS-CoV-2 on olfactory function, with a significant number of patients reporting anosmia as a symptom. However, our knowledge and understanding of the virus's complete impact on the nose remains poor. This report describes a unique patient case to demonstrate how COVID-19 may be associated with rhinoplasty complications such as septal perforation.

Case Report This is a case report of a previously healthy patient who underwent septorhinoplasty in 2018. She had frequent follow-up including intranasal examinations without evidence of the septal perforation for the 2 years following her operation. In March 2020, the patient was noted to have symptoms suggestive of COVID-19, but testing was not recommended by the pediatrician. Soon after her symptoms resolved, she experienced a sudden onset of whistling and physical examination revealed a perforation in the septum which rapidly enlarged over the ensuing weeks. She tested positive for the COVID-19 antibody. After confirming that she no longer had an active infection via antigen testing, she underwent repair of her septal perforation without complications.

Conclusion This case report illustrates a septorhinoplasty complication that may be associated with COVID-19.
\end{abstract}

Corinne Wee

weecorinne@gmail.com

Bahman Guyuron

bahman.guyuron@gmail.com

1 Department of Plastic Surgery, Case Western Reserve University, Cleveland, OH, USA

2 The Zeeba Clinic, 29017 Cedar Rd, Cleveland, OH, USA
Further study into this virus's impact on vascularity and wound healing, specifically in the nose, is recommended. Level of Evidence $V$ This journal requires that authors assign a level of evidence to each article. For a full description of these Evidence-Based Medicine ratings, please refer to the Table of Contents or the online Instructions to Authors www.springer.com/00266.

Keywords Septal perforation - Coronavirus - COVID-19 . Rhinoplasty $\cdot$ Septorhinoplasty complication $\cdot$ Wound healing

\section{Introduction}

As our understanding of SARS-Coronavirus-2 (COVID19) continues to evolve, its impact on multiple body systems and anatomical locations is increasingly evident. While largely understood as a virus affecting the lower respiratory tract, its impact throughout the respiratory tract has been established with growing patient reports of olfactory dysfunction. This, along with the effect of COVID-19 on the vascular system, should serve as a warning to surgeons operating on the nose. This case report illustrates the potential COVID-19-related wound complications in a septorhinoplasty patient.

\section{Case Report}

This is a case report of an 18-year-old, previously healthy female who presented with a spontaneous septal perforation 2 years status-post-septorhinoplasty with bilateral inferior turbinectomy. The septorhinoplasty was performed via an open technique with open septal cartilage harvest. 
There were no complications during the procedure, including no disruption of the mucoperichrondrium. This patient and her family are well-known to the practice and she had no history of cardiovascular disease, tobacco or cocaine use. She underwent a routine pre-operative workup, including a basic coagulation profile, which was all within normal limits.

During this patient's first year post-operatively, she had persistent congestion with two nasal cultures positive for Methicillin-sensitive Staphylococcus aureus treated with Bactroban and Bactrim; her symptoms resolved with these medications. Because of this, she was followed closely but was never noted to have any septal perforation or other wound-healing complications on repeated documented intranasal examinations.

In March 2020 (21 months post-op), she developed a sore throat, fatigue, myalgias, and loss of appetite approximately one week after traveling out of state via airplane. COVID-19 testing was limited at the time of her symptoms, and in the absence of fever and need for hospitalization, her pediatrician did not recommend COVID19 testing. Her symptoms persisted for approximately one week, after which they resolved spontaneously. She denied any trauma or illicit drug use in the following months, and did not recall any nasal decongestant use. At 24 months post-op, she noticed a sudden onset of whistling while breathing that prompted her to present to the plastic surgery clinic, where a 2-mm septal perforation was noted. A culture taken at this time grew only mixed skin flora. She was scheduled for repair of her septal perforation. Again, pre-operative workup was within normal limits, except for a positive COVID-19 antibody test. Her COVID antigen test was negative, thus excluding an active infection. In the absence of active infection, we decided to proceed with repair. Approximately 2 weeks later, at the time of surgery, the perforation was noted to have roughly quadrupled in size compared to her exam in the office (Fig. 1). She underwent perforation repair with conchal cartilage

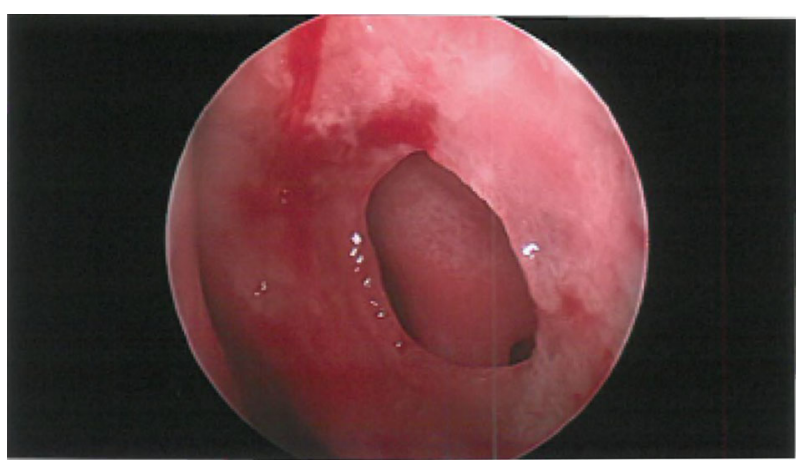

Fig. 1 Septal perforation visualized with endoscopy interposition graft and healed uneventfully with complete closure of the perforation (Fig. 2).

\section{Discussion}

Perhaps one of the most significant findings in COVID-19 patients has been the cardiovascular manifestations, ranging from myocardial infarction and stroke $[1,2]$ to rarer complications such as bilateral radial artery occlusion in a previously healthy patient [3]. The exact mechanism remains unclear. Several theories exist as to the origin of COVID-19-induced vasculopathy. SARS-CoV-2 is known to infect its hosts via ACE2 receptors, which are found in vascular endothelial cells and multiple other organs [4]. A study by Varga et al. supports this theory by identifying COVID-19 virus particles in the endothelial cells of vascular specimens obtained from infected patients [5]. Another theory is that vascular injury may be secondary to cytokine-induced endothelial destruction, analogous to mechanisms exhibited by other viruses such as influenza [6-8]. Despite the need for further research into the pathogenesis for COVID-19-induced vasculopathy, its reported effects on the cardiovascular system have been significant. With reports of cardiovascular morbidity currently focused on major inpatient complications, there is a paucity of literature regarding potential outpatient complications. This could have significant implications for surgical fields, where vascular damage could negatively affect wound healing.

Surgery involving the nose may be especially susceptible to wound healing complications related to COVID-19. Identified as COVID-19's point of entry into the body, the nasopharynx is significantly affected by the virus. Up to $85.6 \%$ of COVID-19 patients report olfactory dysfunction

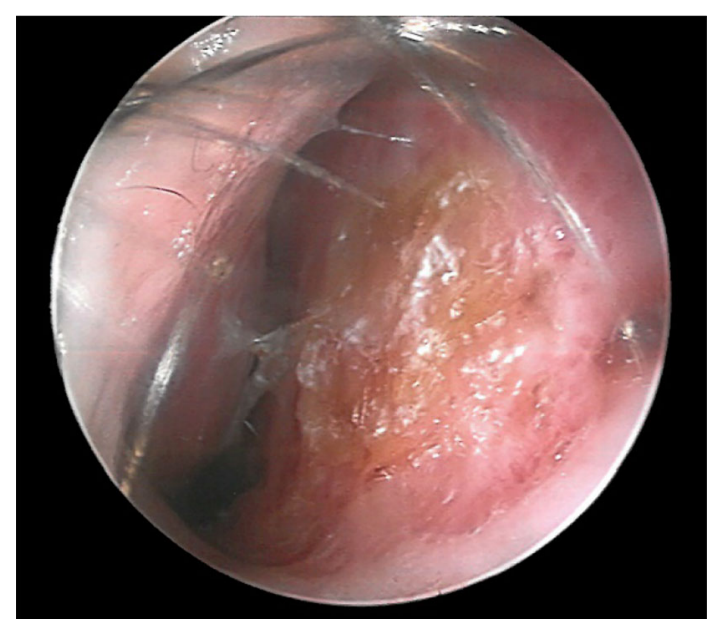

Fig. 2 Septum 4 months following successful septal perforation repair 
[9], with some of these patients reporting post-viral anosmia that is persistent for weeks after infection. Whilst there may be an element of obstructive anosmia (e.g. secondary to mucosal edema and congestion), this prolonged time course suggests that COVID-19 may cause long-term damage to nasal epithelial cells and that anosmia may not resolve until the recovery or regeneration of these cells [10]. In fact, histologic analysis of olfactory epithelial cells after viral infection resulting in anosmia demonstrates a loss of cilia and olfactory sensory neurons [11]. Similar to the mechanism behind COVID-induced vasculopathy, SARS-CoV-2 infects the nasopharynx via ACE2 membrane-bound epithelial receptors. These ACE2 receptors are frequently identified on histologic study of nasal mucosa, revealing the mode of entry of COVID-19 into the nasopharynx [12].

Septoplasty, by virtue of removal of the cartilage and potential alteration in the mucoperichondrial circulation, renders the septum more vulnerable, and perforation can readily be caused by the frequent use of nasal decongestants [13]. The overall rate of septal perforation following septoplasty is quoted to be $2.6-5 \%$ [14, 15], with most cases occurring while damaged tissues are still healing within the first 12 months post-operatively. This patient's rapidly progressive, spontaneous septal perforation two years after surgery (without antecedent trauma and in the absence of other significant risk factors) may be associated with COVID-19.

There are published reports of COVID-19 patients with unusually high incidences of facial skin and oral mucosa injuries $[16,17]$, though a causal mechanism has not been established. Our case report is further limited by the fact that our patient, despite her suggestive symptoms and a positive COVID-19 antibody test, may not have had a symptomatic COVID-19 infection prior to her clinical presentation. It is possible that this patient was an asymptomatic carrier of COVID-19, while being afflicted with another viral illness (e.g. influenza) when she first noticed symptoms of septal perforation at 21 months postop. In addition, we are unsure of the exact onset of her septal perforation and can only use her date of presentation as a timepoint in this report.

Any infection involving the nasopharynx can potentially compromise vascular integrity, but there are no documented cases of delayed septal perforations in the context of other viral infections, with the exception of severely immunocompromised patients [18]. Another possible contributing cause of our patient's septal perforation may be over-the-counter (OTC) nasal decongestant use. However, she did not report regular use of nasal decongestants. Furthermore, at 21 months post-op, there should be minimal risk in inducing wound healing complications with OTC nasal decongestants alone. There has been one documented case of septal perforation associated with rhinitis medicamentosa but this was in the context of continuous, excessive doses of OTC nasal decongestants for several years [19]. It is our assumption that the known, though still-emerging vasculopathic effects of COVID-19 is the most likely primary contributor to this patient's delayed septal perforation. Additional cases and further studies may elucidate specific pathological change in nasal mucosa circulation associated with COVID-19.

\section{Conclusion}

This case represents the first published report of a nasal septal perforation related to COVID-19, possibly due to both its direct effect on the nasopharynx as a site of viral entry and impact on local tissue vascularity. While this patient had previous nasal MSSA infections, nasal culture at the time of perforation did not show any active infection. Furthermore, this patient was young, healthy, without related comorbidities, and over 2 years out from her initial surgery-all factors that would generally deem her very low risk for this complication.

A major limitation is that although this patient had a positive COVID-19 antibody test, she never underwent a COVID-19 PCR test at the time of her initial clinical symptoms. This was unfortunately due to limited quantity of testing at the time of her symptoms. Therefore, it is possible that her symptoms in March were attributable to a different viral infection. While we lack the ability to determine causality for this complication, it is our intention that this case serve as a warning of the potential effects of COVID-19 on the nose.

Considering COVID-19's known capability to invade the nasopharynx and its negative effects on vascularity, COVID-19 may be a risk factor for septal perforation after septorhinoplasty. This case report illustrates the potential impact of COVID-19 on surgical outcomes, particularly in surgery involving the nose. COVID testing policies vary amongst surgery centers. This is certainly a complication of which providers should be mindful, especially as we educate pre-surgical patients on the potential risks of COVID19. It may prove prudent to perform more widespread COVID testing as a part of the pre-surgical evaluation. Further research into impaired wound healing as a possible COVID-19-related complication is indicated.

Funding This study has received an IRB exemption (IRB STUDY20201313).

Compliance with Ethical Standards 
Conflict of interest The authors declare that they have no conflicts of interest to disclose.

Informed Consent For this type of study, informed consent is not required.

\section{References}

1. Yaghi S et al (2020) SARS-CoV-2 and stroke in a New York healthcare system. Stroke 51:2002-2011. https://doi.org/10.1161/ STROKEAHA.120.030335

2. Bandyopadhyay D et al (2020) COVID-19 pandemic: cardiovascular complications and future implications. Am J Cardiovasc Drugs. https://doi.org/10.1007/s40256-020-00420-2

3. Bouwhuis A et al (2020) First, do no harm: bilateral radial artery occlusion in a COVID-19 patient. J Clin Monit Comput. https:// doi.org/10.1007/s10877-020-00555-2

4. Kuba $\mathrm{K}$ et al (2005) A crucial role of angiotensin converting enzyme 2 (ACE2) in SARS coronavirus-induced lung injury. Nat Med 11:875-9. https://doi.org/10.1038/nm1267

5. Varga Z et al (2020) Endothelial cell infection and endotheliitis in COVID-19. Lancet 395:1417-1418. https://doi.org/10.1016/ S0140-6736(20)30937-5

6. Hendren NS et al (2020) Description and proposed management of the acute COVID-19 cardiovascular syndrome. Circulation 141:1903-1914. https://doi.org/10.1161/CIRCULATIONAHA. 120.047349

7. Cotran RS, Pober JS (1989) Effects of cytokines on vascular endothelium: their role in vascular and immune injury. Kidney Int 35:969-75. https://doi.org/10.1038/ki.1989.80

8. Puech-Leao P, Cesar LAM, De Luccia N (2020) COVID-19, vascular diseases, and vascular services. Clinics (Sao Paulo) 75:e1979. https://doi.org/10.6061/clinics/2020/e1979

9. Lechien JR et al (2020) Olfactory and gustatory dysfunctions as a clinical presentation of mild-to-moderate forms of the coronavirus disease (COVID-19): a multicenter European study. Eur
Arch Otorhinolaryngol 277:2251-2261. https://doi.org/10.1007/ s00405-020-05965-1

10. Han AY et al (2020) Anosmia in COVID-19: mechanisms and significance. Chem Senses. https://doi.org/10.1093/chemse/ bjaa040

11. Jafek BW et al (2002) Biopsies of human olfactory epithelium. Chem Senses 27:623-8. https://doi.org/10.1093/chemse/27.7.623

12. Bertram $S$ et al (2012) Influenza and SARS-coronavirus activating proteases TMPRSS2 and HAT are expressed at multiple sites in human respiratory and gastrointestinal tracts. PLoS One 7:e35876. https://doi.org/10.1371/journal.pone.0035876

13. Dosen LK, Haye R (2007) Nasal septal perforation 1981-2005: changes in etiology, gender and size. BMC Ear, Nose Throat Disord 7:1. https://doi.org/10.1186/1472-6815-7-1

14. Muhammad IA, Nabil-ur R (2003) Complications of the surgery for deviated nasal septum. J Coll Physicians Surg Pak 13:565-8. https://doi.org/10.2003/JCPSP.565568

15. Dabrowska-Bien J et al (2018) Complications in septoplasty based on a large group of 5639 patients. Eur Arch Otorhinolaryngol 275:1789-1794. https://doi.org/10.1007/s00405-0184990-8

16. Tomo S, Miyahara GI, Simonato LE (2020) Oral mucositis in a SARS-CoV-2-infected patient: secondary or truly associated condition? Oral Dis. https://doi.org/10.1111/odi.13570.EPubJul29

17. Singh C, Tay J, Shoqirat N (2020) Skin and mucosal damage in patients diagnosed with COVID-19: a case report. J Wound Ostomy Continence Nurs 47:435-438. https://doi.org/10.1097/ WON.0000000000000690

18. Rejali SD et al (1999) Acquired immune deficiency syndrome (AIDS) presenting as a nasal septal perforation. Rhinology 37:93-5

19. Keyserling HF et al (2006) Nasal septal perforation secondary to rhinitis medicamentosa. Ear Nose Throat J 85(376):378-9

Publisher's Note Springer Nature remains neutral with regard to jurisdictional claims in published maps and institutional affiliations. 